\title{
Concerning Limitations and Further Corrections to Geometric-Optical Theory for LF, VLF Propagation Between the Ionosphere and the Ground ${ }^{1}$
}

\author{
J. Ralph Johler \\ Contribution From the Central Radio Propagation Laboratory, National Bureau of Standards, \\ Boulder, Colo.
}

\begin{abstract}
Considerable use has been made of the geometric-optical propagation theory to describe long wavelength terrestrial radio waves between the ionosphere and the ground by Bremmer [1949], Wait and Murphy [1957], Wait [1961], Wait and Conda [1961], and Johler [1961, [1962]. The physical interpretation of pulse signals propagated around the terrestrial sphere as an application of the theory has been described by Johler [1962, 1963a, 1963b]. Indeed, the use of the Loran-C radio navigation-timing system to study the propagation of LF ionospheric waves has given further impetus to such a study.

The geometric series from which the geometric-optical ray limit is derived like the full wave residue series or the series of zonal harmonics [Johler and Berry, 1962] is rigorous. However, the comparatively gross approximations usually employed in the geometric-optical ray limit are worthy of careful scrutiny at this time since the rigorous residue series methods have been worked out in some detail for large scale computers [Johler and Berry, 1962, 1963]. It is apparent from the rigorous theory that the geometric-optical rays do not merely diffract around the curvature of the terrestrial sphere but at considerable distance beyond the geometric-optical horizon are built up to stronger fields by additional ionospheric reflections of the nature of a detached mode of propagation not ordinarily taken into account by the saddle point method of the ray limit.
\end{abstract}

\section{Introduction}

The display of time-separated pulses on a low frequency Loran-C radio navigation-timing. oscillogram [Johler, 1962] at considerable distance from the transmitter can be explained by the geometric-optical rays, figure 1 . Thus, the first pulse to arrive at the receiver $(o)$ from the transmitter $(s)$ can be considered as a signal propagated via the ground wave $(j=0)$ over the surface of the ground with which the geodetic $d=a \theta$ is associated (the waves in the earth are completely absorbed and hence do not ordinarily emerge again). Another pulse will arrive 20 to $40 \mu$ sec later by propagating over the ray $(j=1)$ as a single ionospheric reflection. Higher $\operatorname{order}(j=2,3,4 \ldots)$ pulses will arrive at the receiver $(0)$ by propagation over rays which reflect two or more times between the ground and the ionosphere, arriving at the receiver at ever later times. These pulses are quite calculable for measured models for the ionosphere [Johler, 1963a and 1963b] from the Fourier transform-integral theorem, employing the theory of propagation for either the geometric-optical series or the more conventional residue (mode) series. Concerning the latter, Bremmer [1949] in his well-known textbook is quoted, "We begin by remarking that for the skywave the development of the residue method is, in general, less clear than the geometric-optical method . . . ." Indeed, the geometric-optical method provides a clear physical interpretation of the rays without the pulse solution, where, on the other hand, the residue or mode theory does not split into time-separated pulses without the Fourier inversion to the time domain. Thus, the terms of the residue series (modes) are not clear as far as a physical interpretation of the time domain is concerned. Both methods of course should yield identical numerical values, but the approximations used in the geometricoptical ray limit of the rigorous series are subject of scrutiny in this paper by a comparison with the rigorous residue or zonal harmonics methods. 


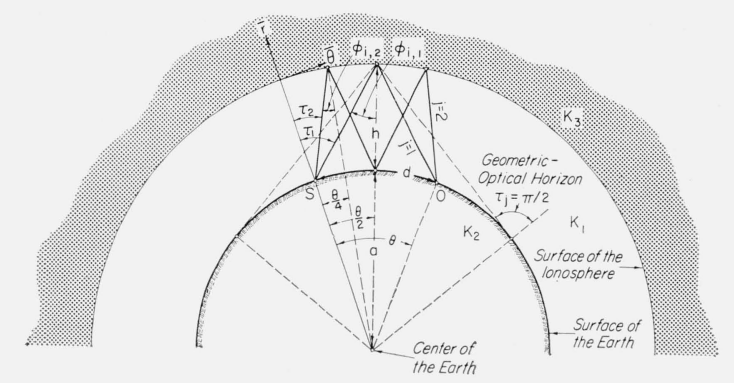

Figure 1. Diagrammatic illustration of the geometric optical rays in the ground-ionosphere guide.

\section{Theory of the Geometric-Optical Series}

The propagation problem described, figure 1, has been formulated rigorously for a source $s$ and an observer $o$, in the guide between the ionosphere and ground without regard to geometricoptical rays, in a companion paper, Johler and Berry [1963]. This solution is the series of zonal harmonics, which for the particular case of a vertical electric field, $E_{r}$, volts/meter, figure 1 , assuming a source dipole current-moment, $I_{0} l=4 \pi / \mu_{0} c \sim 3.38\left(10^{-2}\right)$ ampere-meters,

$$
\begin{gathered}
E_{r}=\frac{1}{k_{1}^{2} a^{4}} \sum_{n=0}^{\infty} n(n+1)(2 n+1) P_{n}(\cos \theta) \zeta_{n}^{(2)}\left(k_{1} a\right) \psi_{n}\left(k_{1} a\right) \\
\times\left\{1+R_{n} \frac{-\zeta_{n}^{(2)}\left(k_{1} a\right)}{\psi_{n}\left(k_{1} a\right)}\right\} \quad\left\{1+T_{n} \frac{-\psi_{n}\left(k_{1} a\right)}{\zeta_{n}^{(2)}\left(k_{1} a\right)}\right\}\left\{1-R_{n} T_{n}\right\}^{-1}, \quad(n=0,1,2,3 \ldots)
\end{gathered}
$$

$k_{1}$ and $a$ are constants, $k_{1}=\frac{\omega}{c} \eta_{1} \cong \frac{\omega}{c}, a$ is the radius of the terrestrial sphere at a frequency $f=\omega / 2 \pi$ where $c$ is the speed of light. $\quad P_{n}(z)$ is the solution of Legendre's differential equation,

$$
\left(1-z^{2}\right) \frac{d^{2} P_{n}(z)}{d z^{2}}-2 z \frac{d P_{n}(z)}{d z}+n(n+1) P_{n}(z)=0
$$

Also,

$$
\begin{gathered}
\psi_{n}(z)=\sqrt{\frac{\pi z}{2}} J_{n+1 / 2}(z), \\
\zeta_{n}^{(2)}(z)=\sqrt{\frac{\pi z}{2}} H_{n+1 / 2}^{(2)}(z),
\end{gathered}
$$

where $J_{n+1 / 2}(z)$ and $H_{n+1 / 2}^{(2)}(z)$ are Bessel and Hankel functions of order $n+1 / 2$ and argument $(z)$ and the Hankel function $H_{n}^{(2)}$ is of the second kind. The quantities $R_{n}$ and $T_{n}$ are related to the spherical reflection coefficients, $R_{n}^{s}$ and $T_{n}^{s}$, respectively,

where

$$
\begin{aligned}
& R_{n}=\frac{-\psi_{n}\left(k_{1} a\right)}{\zeta_{n}^{(2)}\left(k_{1} a\right)} R_{n}^{s}, \\
& T_{n}=\frac{-\zeta_{n}^{(2)}\left(k_{1} d\right)}{\psi_{n}\left(k_{1} d\right)} T_{n}^{s},
\end{aligned}
$$

$$
R_{n}^{s}=\frac{\frac{1}{k_{1}} \frac{\psi_{n}^{\prime}\left(k_{1} a\right)}{\psi_{n}\left(k_{1} a\right)}-\frac{1}{k_{2}} \frac{\psi_{n}^{\prime}\left(k_{2} a\right)}{\psi_{n}\left(k_{2} a\right)}}{-\frac{1}{k_{1}} \frac{\zeta_{n}^{(2) \prime}\left(k_{1} a\right)}{\zeta_{n}^{(2)}\left(k_{1} a\right)}+\frac{1}{k_{2}} \frac{\psi_{n}^{\prime}\left(k_{2} a\right)}{\psi_{n}\left(k_{2} a\right)}},
$$




$$
T_{n}^{s}=\frac{\frac{1}{k_{1}} \frac{\zeta_{n}^{(2)}{ }^{\prime}\left(k_{1} d\right)}{\zeta_{n}^{(2)}\left(k_{1} d\right)}-\frac{1}{k_{3}} \frac{\zeta_{n}^{(2) '}\left(k_{3} d\right)}{\zeta_{n}^{(2)}\left(k_{3} d\right)}}{-\frac{1}{k_{1}} \frac{\psi_{n}^{\prime}\left(k_{1} d\right)}{\psi_{n}\left(k_{1} d\right)}+\frac{1}{k_{3}} \frac{\zeta_{n}^{(2) \prime}\left(k_{3} d\right)}{\zeta_{n}^{(2)}\left(k_{3} d\right)}}
$$

where $d=a+h$ and $h$ is the height of the ionosphere boundary above the surface and

$$
k_{3}=\frac{\omega}{c} \sqrt{1-i \frac{\omega_{N}^{2}}{\omega(\nu+i \omega)}}
$$

for a plasma frequency squared $\omega_{N}^{2}$ and a collision frequency $\nu$

The denominator in (1) is expanded in the geometric-optical series [Bremmer, 1949],

$$
\left\{1-R_{n} T_{n}\right\}^{-1}=1+\sum_{j=1}^{\infty} R_{n}^{j} T_{n}^{j},
$$

provided $\left|R_{n}^{j} T_{n}^{j}\right|<1$, and the field $E_{r}$ is expressed in the geometric-optical series,

$$
E_{r}=E_{r, 0}+\sum_{j=1}^{\infty} E_{r, j}
$$

where, the ground wave, $E_{r, 0}$, which can be regarded as the zero order $(j=0)$ term of the series,

$$
E_{r, 0}=\frac{1}{k_{1}^{2} a^{4}} \sum_{n=0}^{\infty} n(n+1)(2 n+1) P_{n}(\cos \theta) \zeta_{n}^{(2)}\left(k_{1} a\right) \psi_{n}\left(k_{1} a\right)\left\{1+R_{n}^{s}\right\}
$$

and a particular ionospheric wave, $j$,

$$
\begin{aligned}
E_{r, j}=\frac{1}{k_{1}^{2} a^{4}} \sum_{n=0}^{\infty} n(n+1)(2 n+1) P_{n}(\cos \theta)\left\{1+R_{n}^{s}\right\}^{2} & \left\{\frac{-\psi_{n}\left(k_{1} a\right)}{\zeta_{n}^{(2)}\left(k_{1} a\right)} \frac{-\zeta_{n}^{(2)}\left(k_{1} d\right)}{\psi_{n}\left(k_{1} d\right)}\right\}^{j} \\
& \times \zeta_{n}^{(2)}\left(k_{1} a\right) \psi_{n}\left(k_{1} a\right)\left\{R_{n}^{s}\right\}^{j-1}\left\{T_{n}^{s}\right\}^{j} .
\end{aligned}
$$

The expressions (12) and (13) can be evaluated directly at long wavelengths ( $<30 \mathrm{kc} / \mathrm{s})$ by summing the slowly converging series according to methods described by Johler and Berry [1962].

On the other hand, highly convergent representation of (12) and (13) can be obtained from the contour integrals,

$$
\begin{gathered}
E_{r, 0}=\frac{i}{k_{1}^{2} a^{4}} \int_{C} \frac{v\left(v^{2}-\frac{1}{4}\right) P_{v-1 / 2}(-\cos \theta)}{\cos v \pi} \zeta_{v-1 / 2}^{(2)}\left(k_{1} a\right) \psi_{v-1 / 2}\left(k_{1} a\right)\left[1+R_{v-1 / 2}^{s}\right] d v \\
E_{r, j}=\frac{i}{k_{1}^{2} a^{4}} \int_{C} \frac{v\left(v^{2}-\frac{1}{4}\right) P_{v-1 / 2}(-\cos \theta)}{\cos v \pi} \zeta_{v-1 / 2}^{(2)}\left(k_{1} a\right) \psi_{v-1 / 2}\left(k_{1} a\right)\left[1+R_{v-1 / 2}^{s}\right]^{2} \\
\left.\times\left\{\frac{-\psi_{v-1 / 2}\left(k_{1} a\right)}{\zeta_{v-1 / 2}^{(2)}\left(k_{1} a\right)} \cdot \frac{-\zeta_{v-1 / 2}^{(2)}\left(k_{1} d\right)}{\psi_{v-1 / 2}\left(k_{1} d\right)}\right\}^{j} R_{v-1 / 2}^{s}\right]^{j-1}\left[T_{v-1 / 2}^{s}\right]^{j} d v
\end{gathered}
$$

where the poles of order $j$ are associated with $T_{v-1 / 2}^{s}$ as the roots of $v=v_{s}$, of (8),

$$
-\frac{1}{k_{1}} \frac{\psi_{v-1 / 2}^{\prime}\left(k_{1} d\right)}{\psi_{v-1 / 2}\left(k_{1} d\right)}+\frac{1}{k_{3}} \frac{\zeta_{v-1 / 2}^{(2)}\left(k_{3} d\right)}{\zeta_{v-1 / 2}^{(2)}\left(k_{3} d\right)}=0 .
$$

Integral (14) contains the real axis poles, $v=n-\frac{1}{2}=\frac{1}{2}, \frac{3}{2}, \frac{5}{2}$. . ., of the original series of zonal harmonics; and the only other poles of order $(j+1)$ are those of $R_{v-1 / 2}^{s}$, or, eq (8), the roots $v=v_{s}$ of

$$
D_{v-1 / 2}=-\frac{1}{k_{1}} \frac{\zeta_{v-1 / 2}^{(2)}\left(k_{1} a\right)}{\zeta_{v-1 / 2}^{(2)}\left(k_{1} a\right)}+\frac{1}{k_{2}} \frac{\psi_{v-1 / 2}^{\prime}\left(k_{2} a\right)}{\psi_{v-1 / 2}\left(k_{2} a\right)}=0
$$


Equations (16) and (17) are readily evaluated by methods of Johler and Berry [1963] in a companion paper. The convergence of the integrals (14) and (15) is assured for $n=v-\frac{1}{2}$ in $(10)$ by $\left|R_{v-1 / 2}^{j} T_{v-1 / 2}^{j}\right|<1$, which permits interchange of order of integration $\int_{c}$ and summation $\sum_{j}$ since the series converges uniformly with the circle of convergence, $\left|R_{v-1 / 2} T_{v-1 / 2}\right|=1$.

Employing the approximations,

$$
v_{s} \cong\left(k_{1} a\right)+\left(k_{1} a\right)^{1 / 3} \tau_{s},
$$

with approximations of Debye or Hankel approximation [Johler and Berry, 1963; Berry, 1963], which substitutes Hankel functions of order $1 / 3$ for those of complex order, Bremmer [1949] has introduced a determination of the roots $\tau=\tau_{s}, v=v_{s}$ of (7) as the roots of Riccati's differential equation,

$$
\frac{d \delta}{d \tau}-2 \delta^{2} \tau+1=0
$$

which, incidentally, have been tabulated [Johler, Walters, and Lilley, 1959], where for vertical polarization, $\delta=\delta_{e}$,

$$
\delta_{e}=\left\{-i k_{2}^{2} \alpha^{1 / 3} k_{1}^{2}\right\}\left\{\left(k_{1} a\right)^{1 / 3} \sqrt{\left(k_{3}^{2} / k_{1}^{2}\right)-1}\right\}^{-1} .
$$

However, as has been demonstrated in previous papers, Johler and Berry [1962] (17) can be evaluated directly. The integral (14) then becomes the classical series of residues, upon closing the contour along the imaginary axis of the complex $v$-plane with the right half of the $v$-plane,

$E_{r, 0}=\frac{-2 \pi}{k_{1}^{2} a^{4}} \sum_{s=1}^{\infty} \frac{v_{s}\left(v_{s}^{2}-\frac{1}{4}\right) P_{v_{s}-1 / 2}(-\cos \theta)}{\cos v_{s} \pi} \zeta_{v_{s}^{-1 / 2}}^{(2)}\left(k_{1} a\right) \psi_{v_{s}-1 / 2}\left(k_{1} a\right) \times \frac{\eta_{v_{s}-1 / 2}}{\frac{\partial}{\partial v}\left\{D_{v-1 / 2}\right\}_{v=v_{s}}}+\frac{i}{k_{1}^{2} a^{4}} \int_{-i^{\infty}}^{i^{\infty}} f(v) d v$,

where $R_{v-1 / 2}=\eta_{v-1 / 2} / D_{v-1 / 2}$. This is the well-known residue series, representing the first term or "pure" groundwave term of the geometric-optical series. The line integral has been found to be negligible by Berry [1963] in a companion paper. Computation details for this residue series (21) and the zonal harmonics series (12) have been detailed, Johler and Berry [1962]. The evaluation of the integral (21) was carried out in a companion paper by Johler and Berry [1963].

The zonal harmonics series before splitting into a geometric-optical series can also be transformed,

$$
\begin{aligned}
E_{r}=\frac{i}{k_{1}^{2} a^{4}} \int_{e} \frac{v\left(v^{2}-\frac{1}{4}\right) P_{v-1 / 2}(-\cos \theta)}{\cos v \pi} \zeta_{v-1 / 2}^{(2)}\left(k_{1} a\right) \psi_{v-1 / 2}\left(k_{1} a\right)\left\{1+R_{v-1 / 2}^{s}\right\} \\
\left.\qquad 1+T_{v-1 / 2}^{s} \frac{-\zeta_{v-1 / 2}^{(2)}\left(k_{1} d\right)}{\psi_{v-1 / 2}\left(k_{1} d\right)} \frac{-\psi_{v-1 / 2}\left(k_{1} a\right)}{\zeta_{v-1 / 2}^{(2)}\left(k_{1} a\right)}\right\}\left\{1-\frac{\zeta_{v-1 / 2}^{(2)}\left(k_{1} d\right)}{\psi_{v-1 / 2}\left(k_{1} d\right)} \frac{\psi_{v-1 / 2}\left(k_{1} a\right)}{\zeta_{v-1 / 2}^{(2)}\left(k_{1} a\right)} R_{v-1 / 2}^{s} T_{v-1 / 2}^{s}\right\}^{-1} d v
\end{aligned}
$$

where the integration to be considered is performed in the complex $v$-plane.

Equation (15) represents the ionosphere waves. Indeed, the sum of these waves,

$$
\begin{aligned}
\sum_{i=1}^{\infty} E_{r j}=\frac{i}{k_{1}^{2} a^{4}} \int_{C} \frac{v\left(v^{2}-\frac{1}{2}\right) P_{v-1 / 2}(-\cos \theta)}{\cos v \pi}\left\{\psi_{v-1 / 2}\left(k_{1} a\right)\right\}^{2}\left\{1+R_{v-1 / 2}^{s}\right\}^{2} \\
\times \frac{1}{T_{v-1 / 2}^{s}}\left[\frac{-\psi_{v-1 / 2}\left(k_{1} d\right)}{\zeta_{v-1 / 2}^{(2)}\left(k_{1} d\right)}\right]-R_{r-1 / 2}^{s}\left[\frac{-\psi_{v-1 / 2}\left(k_{1} a\right)}{\zeta_{v-1 / 2}^{(2)}\left(k_{1} a\right)}\right]^{-1} d v,
\end{aligned}
$$

in which the poles of $R_{v-1 / 2}^{s}=\eta_{v-1 / 2} / D_{v-1 / 2}, D_{v-1 / 2}=0$ produce a residue series which is precisely the 
negative of the groundwave. Thus, the residue series resulting from the zeros $v=v_{s}$ of

$$
\frac{1}{T_{v-1 / 2}^{s}}\left[\frac{-\psi_{v-1 / 2}\left(k_{1} d\right)}{\zeta_{r-1 / 2}^{(2)}\left(k_{1} d\right)}\right]-R_{v-1 / 2}^{s}\left[\frac{-\psi_{v-1 / 2}\left(k_{1} a\right)}{\zeta_{v-1 / 2}^{(2)}\left(k_{1} a\right)}\right]=0 .
$$

again yields the total field-ground and ionospheric wave.

It should be quite possible to evaluate (15) for each ionospheric wave, $j=1,2,3$. . ., as a series of residues $v=v_{s}$ for the poles of $R_{v-1 / 2}^{s}=\eta_{v-1 / 2} / D_{v-1 / 2}$ and $T_{v-1 / 2}^{s}=\eta_{v-1 / 2}^{1} / D_{v-1 / 2}^{1}$ for $D_{v-1 / 2}=0$. But, these are not simple poles, hence the application of the Cauchy residue theorem becomes quite complicated. The first ionospheric wave is of particular interest or (23) is written as a series of residues for $j=1$, employing the integration contours used in (21),

$$
\begin{aligned}
& E_{r, 1}=\frac{-2 \pi}{k_{1}^{2} a^{4}} \sum_{s=1}^{\infty} \frac{v_{s}\left(v_{s}^{2}-\frac{1}{4}\right) P_{v_{s}-1 / 2}(-\cos \theta)}{\cos v_{s} \pi} \zeta_{v_{s}^{-1 / 2}}^{(2)}\left(k_{1} a\right) \psi_{n_{s}-1 / 2}\left(k_{1} a\right) T_{v_{s}-1 / 2}^{s} \\
& \times\left\{\frac{2 \eta_{v-1 / 2} \frac{\partial}{\partial v} D_{v-1 / 2}\left[\frac{\partial}{\partial v} D_{v-1 / 2}+\frac{\partial}{\partial v} \eta_{v-1 / 2}\right]-\eta_{v-1 / 2}^{2}\left[\frac{\partial^{2}}{\partial v^{2}} D_{v-1 / 2}\right]}{3\left[\frac{\partial}{\partial v} D_{v-1 / 2}\right]^{3}}\right\}_{v=v_{s}} \\
& +\frac{-2 \pi}{k_{1}^{2} a^{4}} \sum_{s=1}^{\infty} \frac{v_{s}\left(v_{s}^{2}-\frac{1}{4}\right) P_{v_{s}-1 / 2}(-\cos \theta)}{\cos v_{s} \pi} \zeta_{n_{s}-1 / 2}^{(2)}\left(k_{1} a\right) \psi_{v_{s}-1 / 2}\left(k_{1} a\right) \\
& \times\left\{1+R_{v_{s}-1 / 2}^{s}\right\}^{2}\left\{\frac{\eta_{v_{s}-1 / 3}^{1}}{\left\{\frac{\partial}{\partial v} D_{v_{s}-1 / 2}^{1}\right\}_{v=v_{s}}}\right\}
\end{aligned}
$$

where the line integral Berry [1963],

$$
\frac{i}{k_{1}^{2} a^{4}} \int_{-1 \infty}^{1 \infty} f(v) d v
$$

can be neglected. $D_{v-1 / 2}$ is identical with $(17)$ and can be evaluated by previously described methods of Johler [1961] for the first series of (25). The contribution of the second series as a result of the poles $v=v_{s}$ of $D_{v-1 / 2}^{1}=0$, does not occur in Bremmer [1949] and Wait [1961] formulation.

Thus, for example, Bremmer [1949] in his well-known textbook (p. 33 of his document) makes the substitution $\psi_{n}=\frac{1}{2} \zeta_{n}$ (using $\exp (i \omega t)$ time function). This can be accomplished in an exact manner, $\psi_{n}=\frac{1}{2}\left[\zeta_{n}^{(1)}+\zeta_{n}^{(2)}\right]$. This latter exact substitution in (15) results in a single series with only groundwave type poles of importance such as those poles in the first series of (25), and the effect of the ionosphere poles is thrown in essence into the excitation factors, as was accomplished in the less exact geometric-optical theory to be derived subsequently. Detailed study of these matters is reserved for future work.

Thus,

$$
D_{v-1 / 2}^{1}=-\frac{1}{k_{1}} \frac{\psi_{v-1 / 2}^{\prime}\left(k_{1} d\right)}{\psi_{v-1 / 2}\left(k_{1} d\right)}+\frac{1}{k_{3}} \frac{\zeta_{v-1 / 2}^{(2)}\left(k_{3} d\right)}{\zeta_{v-1 / 2}^{(2)}\left(k_{3} d\right)}=0
$$

for $v=v_{s}$ in the second series of (24). Thus, it is unnecessary to make further mathematical development of (25) since techniques for all functions described herein have been described previously, Johler and Berry [1962 and 1963] except for the $\frac{\partial^{2}}{\partial v^{2}} D_{v-1 / 2}$. Since $\frac{\partial}{\partial v} D_{v-1 / 2}$ has been worked out, a continuation of this procedure, although tedious, is tractable. The significance of (25) and (13) is a series of residues and a series of zonal harmonics for the ionospheric geometric-optical wave which, like the ground wave, is rigorous. It is therefore quite tractable but tedious to formulate and calculate rigorous geometric-optical ionospheric waves. This task is reserved for future work. An examination of the more conventional ray limit of the rigorous geometric-optical series will now be made. 
The ray limit of the rigorous geometric-optical series represents an evaluation of the integral (15) employing approximations:

$$
\psi_{v}(z) \cong \exp \left\{\frac{i z}{3}\left\{1-\left(\frac{v}{z}\right)^{2}\right\}^{3 / 2}-\frac{\pi i}{4}\right\}\left\{1-\left(\frac{v}{z}\right)^{2}\right\}^{-1 / 4}
$$

provided $\left|1-\left(\frac{v}{z}\right)^{2}\right|<<1|,| \frac{z}{3}\left\{1-\left(\frac{v}{z}\right)^{2}\right\}^{3 / 2}|>>1|$, where the former condition applies to the low frequency groundwave poles and the latter to the higher frequency case $(100 \mathrm{kc} / \mathrm{s}), z=k, r$ large. Also,

$$
\begin{gathered}
\zeta_{v}^{(2)}(z) \cong \exp \left\{-\frac{i z}{3}\left\{1-\left(\frac{v}{z}\right)^{2}\right\}^{3 / 2}+\frac{\pi i}{4}\right\}\left\{1-\left(\frac{v}{z}\right)^{2}\right\}^{-1 / 4} \\
\frac{\psi_{v}(z)}{\zeta_{v}^{(2)}(z)} \approx \exp \left\{\frac{2 i z}{3}\left\{1-\left(\frac{v}{z}\right)^{2}\right\}^{3 / 2}-\frac{\pi i}{2}\right\} \\
\frac{v\left(v^{2}-\frac{1}{4}\right) P_{v-1 / 2}(-\cos \theta)}{\cos v \pi} \cong 2 \frac{\sqrt{v}\left(v^{2}-\frac{1}{4}\right)}{\sqrt{2 \pi \sin \theta}} \exp \left\{-i v \theta-i \frac{\pi}{4}\right\}
\end{gathered}
$$

for $\operatorname{Im} v$ large or, say, $\operatorname{Im} v>2$, since $v$ is exponential order. Noting the elimination of the poles of the integrand due to $\cos v \pi$ on the real axis as a result of the approximation (30), (15) can be written, $j=1,2,3 \ldots$ or $j \neq 0$,

$$
\begin{aligned}
E_{r, i}=\frac{\exp \left\{i \pi\left(2 j+\frac{1}{4}\right)\right\}}{k_{1}^{2} a^{4} \sqrt{2 \pi \sin \theta}} \int_{-\infty}^{\infty} \frac{\sqrt{v}\left(v^{2}-\frac{1}{4}\right)}{\sqrt{1-\left(\frac{v-\frac{1}{2}}{k_{1} a}\right)^{2}}\left\{1+R_{v-1 / 2}^{s}\right\}^{2}\left\{R_{v-1 / 2}^{s}\right\}^{j-1}\left\{T_{v-1 / 2}\right\}^{j}} \\
\quad \times \exp \left\{-i v \theta-\frac{2}{3} i j\left[k_{1} d\left\{1-\left(\frac{v-\frac{1}{2}}{k_{1} d}\right)^{2}\right\}^{3 / 2}-k_{1} a\left\{1-\left(\frac{v-\frac{1}{2}}{k_{1} a}\right)^{2}\right\}^{3 / 2}\right]\right\} d v .
\end{aligned}
$$

It is common practice to evaluate this integral by the saddle point method, Wait [1961], Bremmer [1949],

$$
\int_{-\infty}^{\infty} A(v) \exp \{f(v)\} d v \simeq \sqrt{\frac{2 \pi}{-f^{\prime \prime}\left(v_{j}\right)}} A\left(v_{j}\right) \exp \left\{f\left(v_{j}\right)\right\}
$$

where $v_{j}$ are the roots, $v=v_{j}$ of $f^{\prime}(v)=0$, or

or

$$
f^{\prime}(v)=\frac{\partial}{\partial v}\left\{-i v \theta-\frac{2 i j}{3}\left[k_{1} d\left\{1-\left(\frac{v-\frac{1}{2}}{k_{1} d}\right)^{2}\right\}^{3 / 2}-k_{1} a\left\{1-\left(\frac{v-\frac{1}{2}}{k_{1} a}\right)^{2}\right\}^{3 / 2}\right]\right\}=0
$$

and

$$
\theta=2 j\left\{\left(\frac{v-\frac{1}{2}}{k_{1} d}\right) \sqrt{1-\left(\frac{v-\frac{1}{2}}{k_{1} d}\right)^{2}}-\left(\frac{v-\frac{1}{2}}{k_{1} a}\right) \sqrt{1-\left(\frac{v-\frac{1}{2}}{k_{1} a}\right)^{2}}\right\}_{v=v_{j}}
$$

$$
f^{\prime \prime}(v)=-2 j\left\{\frac{v-\frac{1}{2}}{\left(k_{1} d\right)^{2}}\left[1-\left(\frac{v-\frac{1}{2}}{k_{1} d}\right)^{2}\right]^{-1 / 2}-\frac{v-\frac{1}{2}}{\left(k_{1} a\right)^{2}}\left[1-\left(\frac{v-\frac{1}{2}}{k_{1} a}\right)^{2}\right]^{-1 / 2}\right\} \text {. }
$$

This leads to the standard geometric-optical formula, Johler [1961], using $t_{j}^{\prime}=t_{j}-D_{j} / c$ where for the ground wave, $j=0, t_{0}^{\prime}=t-\eta_{1} d / c$,

where

$$
E_{r, j}=i \omega d D_{j}^{-1} C \exp \left(i \omega t_{j}^{\prime}\right) G_{j}^{t} G_{j}^{r} \alpha_{j} F_{j} C_{j}
$$

and

$$
D_{j} \simeq 2 j\left[(a+h) \cos \varphi_{i, j}-a \cos \tau_{j}\right],
$$

$$
F_{j}=F_{j}^{t} F_{j}^{r}=\left[F_{j}^{t, r}\right]^{2}=\left[1+R_{v_{j}-y_{2}}\right]^{2}=[1+R]^{2}
$$


for transmitter $(t)$ and receiver $(r)$ over identical ground,

$$
C_{j}=\left[R_{v_{j}-1 / 2}\right]^{j-1}\left[T_{v_{i-1 / 2}}\right]^{j}=[R]^{j-1}[T]^{j}
$$

where $R$ and $T$ are interpreted as the well-known Fresnel reflection coefficients for vertical polarization $(e)$,

$$
\begin{gathered}
R=R_{e}=\frac{\frac{k_{2}}{k_{1}} \cos \tau_{j}-\sqrt{\frac{k_{2}}{k_{1}}-\sin ^{2} \tau_{j}}}{\frac{k_{2}}{k_{1}} \cos \tau_{j}+\sqrt{\frac{k_{2}}{k_{1}}-\sin ^{2} \tau_{j}}}, \\
T=T_{e}=\frac{\frac{k_{3}}{k_{1}} \cos \varphi_{i, j}-\sqrt{\frac{k_{3}}{k_{1}}-\sin ^{2} \varphi_{i, j}}}{\frac{k_{3}}{k_{1}} \cos \varphi_{i, j}+\sqrt{\frac{k_{3}}{k_{1}}-\sin ^{2} \varphi_{i, j}}} .
\end{gathered}
$$

The effect of anisotropy and the variation of electron density and collision frequency with altitude (nonsharply bounded ionosphere) are accounted for by the factor $C_{j}$ which involves the reflection coefficients $R, T$. In the anisotropic case [Johler and Harper, 1962; Johler, 1962], $R$ is replaced by $R_{e}$ and $R_{m}$, the vertical electric and vertical magnetic reflection coefficients, and $T$ is replaced by $T_{e e}, T_{e m}, T_{m e}, T_{m m}$, where $T_{e e}$ is a vertical electric incident wave with a corresponding vertical electric reflected wave, $T_{e m}$ is the abnormal component or vertical magnetic resulting from the vertical electric field at the anisotropic boundary. Similarly, $T_{m m}$ refers to the vertical magnetic incident and reflected and again $T_{m e}$ is a corresponding abnormal component. Thus, for the anisotropic case, Bremmer [1949],

where

$$
C_{j}=\frac{1}{j ! R_{e}} \frac{d^{j}}{d x^{j}}\left[\frac{1+A_{1} x}{1-A_{2} x-A_{3} x^{2}}\right]_{x=0}
$$

$$
\begin{aligned}
& A_{1}=R_{m} T_{m m} \\
& A_{2}=R_{e} T_{e e}+R_{m} T_{m m} \\
& A_{3}=R_{e} R_{m}\left[-T_{e e} T_{m m}+T_{e m} T_{m e}\right] .
\end{aligned}
$$

Explicit expressions for $C_{j}$ are given by Johler [1961]. Johler and Harper [1962], Johler [1962] have devised computation boundaries to evaluate electron density-altitude, $N(h)$ collision frequency-altitude $\nu(h)$ profiles of the lower ionosphere. Also, monoenergetic electron-ion collision frequencies have been introduced into these reflection coefficients, Johler and Harper [1962b].

$$
G_{j}^{t} G_{j}^{r}=G^{2} \cong \sin ^{2} \tau_{j}
$$

for identical transmitter and receiver dipoles, $\mathrm{C}=10^{-7} I_{0} l / d$, where $d=a \theta$, the geodetic, $t_{j}^{\prime}=t-$ $\eta_{1} D_{j} / c\left(\eta_{1} \sim 1\right)$ and

$$
\alpha_{j} \cong \frac{a+h}{a}\left[\frac{2 j \sin \frac{\theta}{2 j}}{\sin \theta}\right]^{\frac{1}{2}}\left[\frac{(a+h)-a \cos \frac{\theta}{2 j}}{(a+h) \cos \frac{\theta}{2 j}-a}\right]_{\left(A_{j}=1\right)}^{\frac{1}{2}} A_{j}
$$

The factor $\alpha_{j}$ is corrected in the caustic region, $\tau_{j} \approx \frac{\pi}{2}$, with the factor $A_{j} \neq 1$, Wait [1960]

$$
A_{j} \cong \sqrt{\frac{\pi z_{j}}{2}} H_{1 / 3}^{(2)}\left(z_{j}\right) \exp \left\{-i\left[5 \pi / 12-z_{j}\right]\right\}
$$

where $z_{j}=k_{1} a \cos ^{3} \tau_{j} / 3 \sin ^{2} \tau_{j}$. Also, in the same region, Wait and Conda [1958] have replaced 
the factor $F=(1+R)^{2}$ with the solution for a plane wave wrapped around an infinitely long: cylinder,

$$
F_{j}^{t, r} \cong \pi^{-1 / 2} \exp \left[-i k_{1} a \theta^{\prime}\right] \int_{\infty \exp [-i 2 \pi / 3]}^{\infty} \frac{\exp \left[-i\left(k_{1} a / 2\right)^{1 / 3} \theta^{\prime} \rho\right]}{W_{1}^{\prime}(\rho)-q W_{1}(\rho)} d \rho
$$

where $\theta^{\prime}=\left(d-d_{H}\right) / 2 a$ and $d_{H}$ is the length of geodetic from transmitter to horizon, $\tau_{j}=\frac{\pi}{2}$, and,

Also,

$$
q=-i\left(\begin{array}{c}
k_{1} a \\
2
\end{array}\right)^{1 / 3} \frac{k_{1}}{k_{2}} \sqrt{1-\frac{k_{1}^{2}}{k_{2}^{2}}}
$$

$$
\begin{gathered}
W_{1}(\rho)=\exp [-2 \pi i / 3] \sqrt{\pi / 3}(-\rho)^{1 / 2} H_{1 / 3}^{(2)}\left\{\frac{2}{3}(-\rho)^{3 / 2}\right\}, \\
W_{1}^{\prime}(\rho)=\exp [-2 \pi i / 3] \sqrt{\pi / 3} \rho H_{2 / 3}^{(2)}\left\{\frac{2}{3}(-\rho)^{3 / 2}\right\},
\end{gathered}
$$

where

$$
-\frac{\pi}{3} \leq \arg \rho<\frac{5 \pi}{3}
$$

and

$$
\arg (-\rho)=\arg \rho-\pi,
$$

$\arg \rho^{m / m}=\frac{m}{n} \arg \rho$ where $m$ and $n$ are integers, whereupon all functions involved are single valued. This can also be written as a residue series summation,

$$
F_{j}^{t, r} \sim-2 i \sqrt{ } \pi \exp \left[-i k_{1} a \theta^{\prime}\right] \sum_{s=0}^{\infty} \frac{\exp \left[-i\left(k_{1} a\right)^{1 / 3} \theta^{\prime} \tau_{s}\right]}{\left[2^{1 / 3} \tau_{s}-q^{2}\right] W_{1}\left(2^{1 / 3} \tau_{s}\right)} .
$$

The use of the cylindrical geodetic in the calculation of the factor $F_{j}^{t, r}$ in (44) can be replaced by the exact solution for a plane wave on a sphere using either the series of zonal harmonics or the residue series of Johler and Berry $[1962,1963]$ or $E_{r}=E_{r 0}(12)$, (14), where $E_{0}$ is the primary field

$$
\operatorname{Lim}_{\mathrm{b} \rightarrow \infty} \frac{E_{r}}{E_{0}}=F_{j}^{t, r} .
$$

\section{Discussion}

The advantages of using geometric-optical theory to interpret pulses in the time domain and to introduce local changes in the reflection processes warrants an investigation to determine corrections or extensions of the theory to make the accuracy of the predicted fields correspond with the same degree as the accuracy of the residue methods. To this end the computation is studied. The ground wave $E_{r, 0}$ portion of the conventional geometric-optics is calculated by methods developed by Bremmer. The formulation of Bremmer's [1949] theory for the large scale electronic computer was presented by Johler, Kellar, and Walters [1956]. A comparison of this method of computation, which employs some approximations, with the rigorous solution originally proposed by Watson [Johler and Berry, 1963] using residue methods and also using the series of zonal harmonics gave identical results.

This leads one to inquire as to how well the higher order terms $j=1,2,3$. . . of the geometric-optical series (36), or indeed, the sum of all significant terms of the series compares with the rigorous solution (23) (25) (16). Employing the computation methods described in the companion paper by Johler and Berry [1963], figures 2, 3, 4 illustrate such a comparison at $100 \mathrm{kc} / \mathrm{s}, 30 \mathrm{kc} / \mathrm{s}$, and $10 \mathrm{kc} / \mathrm{s}$ as a function of distance for various situations. A sharply bounded, isotropic, model ionosphere was employed for this purpose. At $100 \mathrm{kc} / \mathrm{s}$, the geometric-opitcal ray series clings quite accurately to the rigorous curves at short distances, $<400$ $\mathrm{km}$, probably because of the dominance of the Bremmer-van der Pol ground wave term in the series. Indeed, as distance is increased, the accuracy is still quite remarkable in spite of the comparatively gross approximations in (36). The ripples caused by interference of ground and ionospheric waves are followed quite accurately out to approximately $1,000 \mathrm{~km}$. Here 


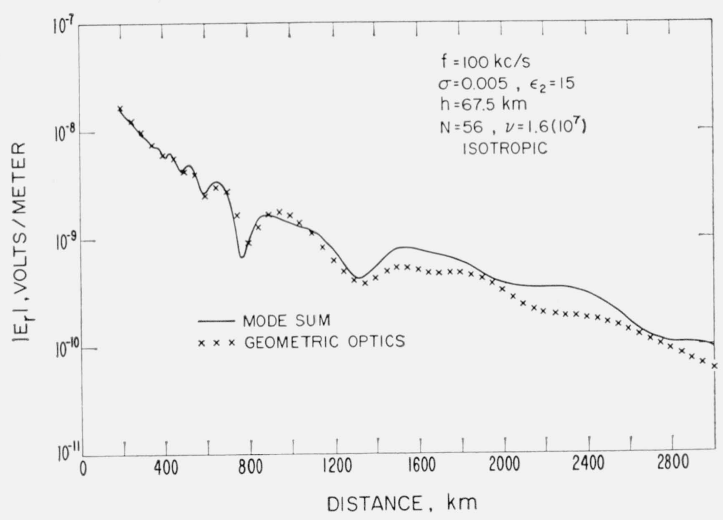

Figure 2. Comparison of the amplitude of the field $\left(\mathrm{E}_{\mathrm{r}}\right)$ as calculated with the rigorous residue series with the geometric-optical ray series approximation $(100 \mathrm{kc} / \mathrm{s})$, including diffractive corrections.

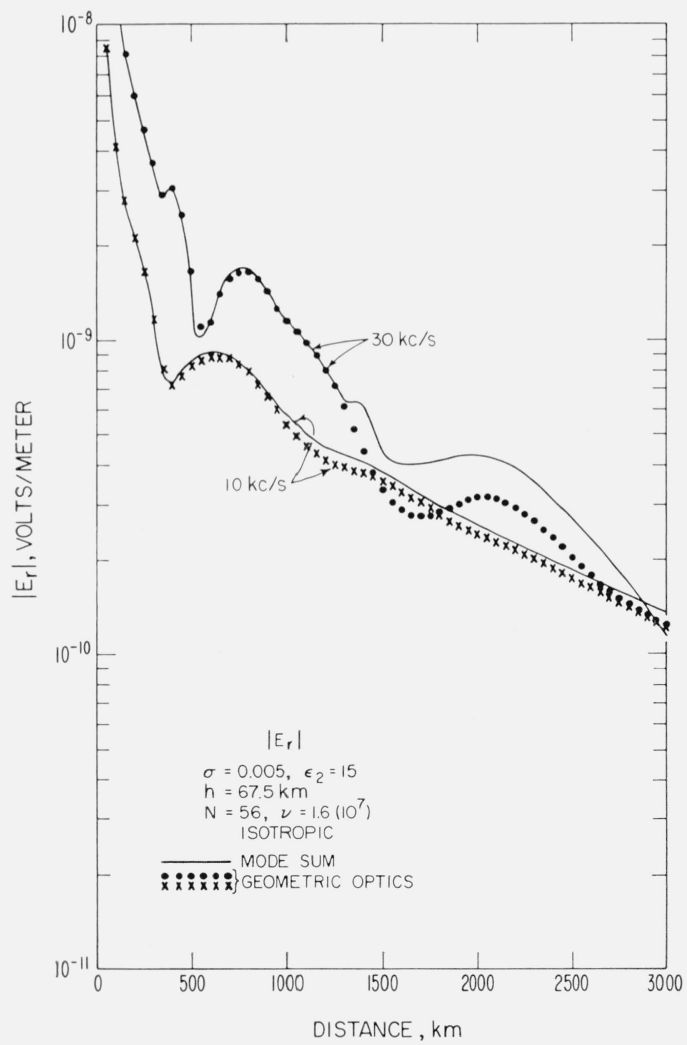

Figure 3. Comparison of the amplitude of the field $\left(\mathrm{E}_{i}\right)$ as calculated with the rigorous residue series with the geometric-optical ray series approximation $(\mathrm{h}=67.5 \mathrm{~km}, 30 \mathrm{kc} / \mathrm{s}, 10 \mathrm{kc} / \mathrm{s})$, including diffractive corrections.

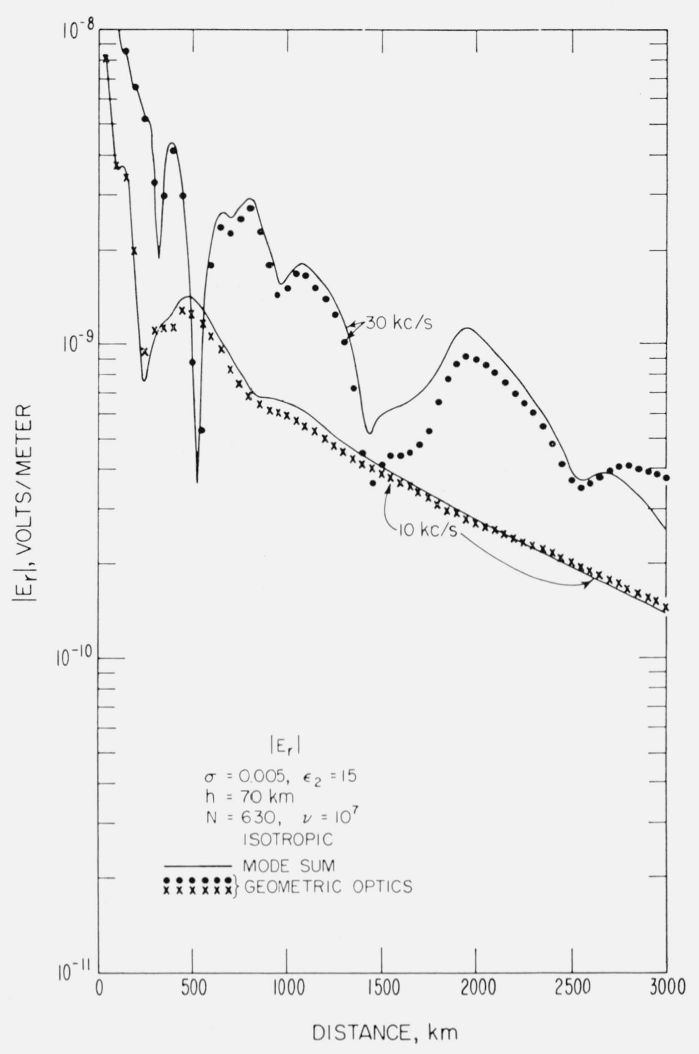

Figure 4. Comparison of the amplitude of the field $\left(\mathrm{E}_{\mathrm{r}}\right)$ as calculated with the rigorous residue series with the geometric-optical ray series approximation $(\mathrm{h}=70 \mathrm{~km}, 30 \mathrm{kc} / \mathrm{s}, 10 \mathrm{kc} / \mathrm{s})$, including diffractive corrections.

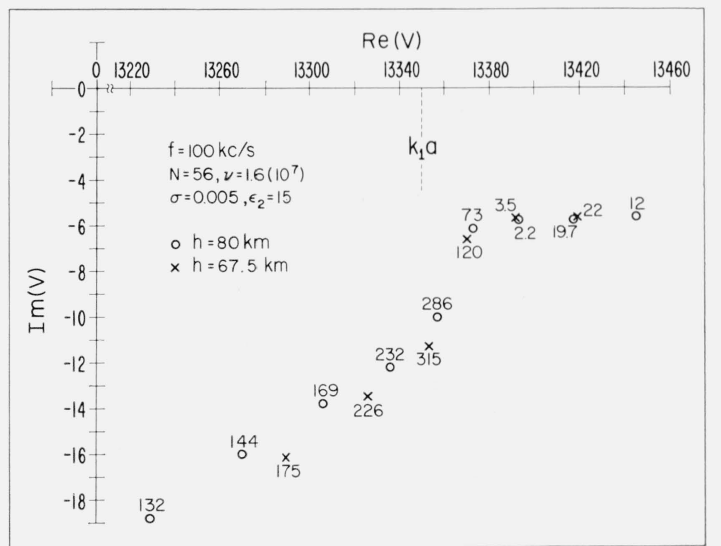

Figure 5. Poles of contour integral in complex $\mathrm{v}$-plane together with excitation factor, illustrating quantization of the propagation. 
also the departure is not serious since no new phenomena are observed in the rigorous theory. The departure does, however, become serious at 2,000 km and greater distance where the field decays more rapidly than is indicated by the rigorous solution. A similar situation is noted, figures 3 and 4 at $30 \mathrm{kc} / \mathrm{s}$ or $10 \mathrm{kc} / \mathrm{s}$. However, at $10 \mathrm{kc} / \mathrm{s}$ it is interesting to note that the serious departure between the geometric-optical and rigorous method is smaller even at distances, say, $2,500 \mathrm{~km}$. This, of course, is contrary to intuition, since it is generally conceded that geometric-optical rays are valid at the high frequency ray limit, $f \rightarrow \infty$. However, the comparison is more subtle, since, the so-called geometric-optical ray theory used at long wavelengths, (36), does employ spherical corrections as described previously. This leads one to inquire as to the cause of the departure of (36) from the rigorous theory and suggests a possible correction to extend the theory to greater distances.

It is possible to question the validity of the approximations for the convergence coefficient, $\alpha_{\mathrm{j}}$, but the calculations have been restricted to distances for which the spherical corrections are valid. The use of the cylindrical approximation (44) of Wait and Conda [1958] to calculate $F_{i}^{t, r}$ could also be questioned. However, a comparison of this factor with the rigorous factor (48) indicates good agreement in the distances of concern, near $\left(d-d_{H}=0\right)$. This leads one to question the saddle point approximation.

The complex $v$-plane of the contour integral (16), representing the rigorous solution to the problem, for which computation details have been presented by Johler and Berry [1963] in a companion paper is illustrated, figure 8 , at a frequency of $100 \mathrm{kc} / \mathrm{s}$. The position of the poles of the integrand are shown graphically, and the excitation factor for each residue is also given. A rather interesting change occurs in the topography of the complex $v$-plane at 100 $\mathrm{kc} / \mathrm{s}$. Thus, at lower frequencies, the poles "march" downward to the left in the fourth quadrant, approaching ever closer to the Im $v$-axis with ever increasing imaginary in a quite regular manner similar to the poles with excitation factor 232, 169, 144, 132, figure 5. However, at $100 \mathrm{kc} / \mathrm{s}$ there is a group of poles in the region $k_{1} a<\operatorname{Re} v<k_{1} d$ which exhibit a sharp discontinuity in this otherwise regular progress, such that the $\operatorname{Im} v$ is comparatively small and the excitation factor is comparatively larger, the residues of the poles with excitation factors $286,232,315,226$, etc., obviously dominate the field at short distances. But, since the $\operatorname{Im} v$ is comparatively large, these modes decrease rapidly with distances and at 1,500 or 2,000 $\mathrm{km}$ no longer dominate. At this point the modes with the somewhat smaller excitation factors, 75, 120, 3.2, 2.2, etc., dominate, since their $\operatorname{Im} v$, which is of exponential order, is considerably smaller. Bremmer [1949] alludes to this quantizing of the field by referring to these latter modes as B-poles, where the former are A-poles. The saddle point approximation apparently implies the A-poles as the dominant field at the surfaces of the earth. It would be necessary to represent the B-poles by rays skirting the ionosphere. Whereas these rays never touch the ground, they fill the space and apparently make a contribution at greater distances, $(>2,000 \mathrm{~km})$.

This suggests evaluating the second summation of (25) as an additive correction to the geometric-optics. Since this residue series involves simple poles for the first ionospheric ray, it is comparatively simple to evaluate by previously described methods, Johler and Berry [1963]. The more complicated first residue series of (25) can also be evaluated for the complete rigorous geometric-optical first ionospheric reflection. It is apparent that a similar rigorous evaluation of the higher order terms of the geometric-optical series $(j=2,3,4$. . ) can be made by similar procedures, the most serious complication being the evaluation of the residues of higher order poles. This is complicated but tractable.

The poles of the second series together with excitation factors ${ }^{2}$ as calculated by (16) are illustrated in table 1 . The poles of the diffraction factors, $R_{v-1 / 2},(17)$, are identical with the groundwave.

2 The excitation factor of the second term-series of (25) is

$$
\left.v_{s}\left(v_{3}^{2}-\frac{1}{4}\right) \zeta_{v_{\infty}-1 / 2}^{(2)}\left(k_{1} a\right) \psi_{v_{s}-1 / 2}\left(k_{1} a\right)\left\{1+R_{v_{s}}^{s}-1 / 2\right\}^{2} \frac{\eta_{v_{s}-1 / 2}^{i}}{\left\{\frac{\partial}{\partial v} D_{v_{s}}^{i}-1 / 2\right.}\right\}_{V=V_{s}}
$$


TABLE 1. Illustrating a comparison of the topography of the $\mathrm{v}$-plane for the ionosphere modes of $\mathrm{D}_{\mathrm{v}-1 / 2}^{\prime}=0$ with mode $B$-poles of $1-\mathrm{R}_{\mathrm{v}-1 / 2} \mathrm{~T}_{\mathrm{v}-1 / 2}=0$

\begin{tabular}{|c|c|c|c|c|c|c|}
\hline \multicolumn{2}{|c|}{$f=100 \mathrm{kc} / \mathrm{s}$} & \multicolumn{2}{|r|}{$k_{1} a=13349.7$} & \multicolumn{3}{|c|}{$h=67.5 \mathrm{~km}$} \\
\hline \multicolumn{4}{|c|}{ Ionosphere poles } & \multicolumn{3}{|c|}{ Mode B-poles } \\
\hline$s$ & $\operatorname{Re} V_{s}$ & $\operatorname{Im} V_{s}$ & |Excitation factor| & $\operatorname{Re} V_{s}$ & $\operatorname{Im} V_{s}$ & |Excitation factor| \\
\hline $\begin{array}{l}1 \\
2 \\
3\end{array}$ & $\begin{array}{l}13418.67 \\
13391.39 \\
13367.29\end{array}$ & $\begin{array}{l}-5.66 i \\
-5.74 i \\
-5.78 i\end{array}$ & $\begin{array}{r}75 \\
5 \\
5\end{array}$ & $\begin{array}{l}13418.17 \\
13391.48 \\
13369.91\end{array}$ & $\begin{array}{l}-5.66 i \\
-5.71 i \\
-6.60 i\end{array}$ & $\begin{array}{r}22 \\
4 \\
126\end{array}$ \\
\hline
\end{tabular}

NoTE: $-i \operatorname{Im} v_{1} \cong-i\left(k_{1} a\right)^{1 / 3} \operatorname{Im} \tau_{0} \cong-15 i$ for ground wave poles (18) which have been tabulated by Johler, Walters, and Lilley [1959].

The ionosphere poles are close to the B-poles of the residue series or mode series representation. However, the excitation factors are different. Note the large excitation factor for $s=1$ of approximately 75 .

Since the ionosphere poles have small Im $v$, these are not negligible at greater distance since the excitation factor is sufficiently large. Indeed, these residues are of sufficient amplitude to compensate or correct the geometric-optical curves, figures 4, 5, where the first and second term of the geometric-optical series are sufficient. Obviously, the corrections for the higher order terms of this series are required at the greater distances $(>\sim 2,000 \mathrm{~km})$. It can then be concluded that the geometric-optical rays are not merely built up by ray diffraction around the terrestrial sphere but indeed are built up by certain ionospheric reflection of the nature of a detached mode. The detailed study of these phenomena is reserved for future work.

\section{Conclusions}

The geometric-optical ray theory, which has been corrected with the conventional spherical corrections, is a reasonably valid theory to distances of approximately 1,500 or 2,000 km. At greater distances and especially at LF $(100 \mathrm{kc} / \mathrm{s})$, it is necessary to correct the field for additional ionospheric propagation, in which case an ionosphere reflection coefficient residue correction can be made. It is quite possible with presently available techniques to evaluate the first term $(j=1)$ of the rigorous geometric-optical series, and the extension to higher order $(j=2,3,4 \ldots)$ terms of the series is tractable but complicated. In any case it is clear that a diffraction theory is not always adequate to describe the field from a geometric-optical point of view, especially at distances greater than $1,500 \mathrm{~km}$. The results of this analysis suggest such extensions for future work.

\section{References}

Berry, L. A. (1964), Some remarks on the Watson transformation and mode theory, J. Res. NBS 68D (Radio Prop.), No. 1, 63.

Bremmeı, H. (1949), Terrestrial radio waves - theory of propagation (Elsevier Publ. Co., Inc., New York, N.Y.).

Johler, J. R. (Oct. 1961), On the analysis of LF ionospheric radio propagation phenomena, J. Res. NBS 65D (Radio Prop.), No. 5, 507-529.

Johler, J. R. (Apr. 1962), Propagation of the low-frequency radio signal, Proc. IRE 50, No. 4, $404-427$.

Johler, J. R. (Sept.-Oct. 1963b), Propagation of the LF radio pulse by a reflection process at the lower ionosphere, J. Res. NBS 6\%D (Radio Prop.), No. 5, 481-499.

Johler, J. R. (Nov. 1963a), Propagation time of a radio pulse, IEEE Trans. PTGAP.

Johler, J. R., and L. A. Berry (Nov.-Dec. 1962), Propagation of terrestrial radio waves of long wavelengththeory of zonal harmonics, J. Res. NBS 66D (Radio Prop.) No. 6, 737-773.

Johler, J. R., and L. A. Berry (1963), On LF/VLF/ELF terrestrial radio waves, Digest of papers, symposium on the ionospheric propagation of VLF radio waves, pp. 43-50 (Boulder, Colo. Aug. 12-14).

Johler, J. R., W. J. Kellar, and L. C. Walters (June 1956), Phase of the low radio frequency ground wave, NBS Cir. 573 (U.S. Govt. Printing Office, Superintendent of Documents, Washington, D.C., 20402).

Johler, J. R., L. C. Walters, and C. M. Lilley (Feb. 1959), Low- and very-low-radio frequency tables of ground wave parameters for the spherical earth theory $i$ the roots of Riccattis differential equation, NBS Technical Note No. 7, PB 151366. 
Wait, J. R. (1960), Diffractive corrections to the geometrical optics of low frequency propagation, Electro magnetic Wave Propagation (Academic Press, London, England).

Wait, J. R. (June 1961), A diffraction theory of LF sky-wave propagation, J. Geophys. Res. 66, No. 6,17131724 .

Wait, J. R. (1963), The mode theory of VLF radio propagation for a spherical earth and a concentric anisotropic ionosphere, Canadian J. Physics 41, 299-315.

Wait, J. R., and A. M. Conda (Oct. 1958), Pattern of an antenna on a curved lossy surface, IRE Trans. on Antennas and Propagation AP-6, 348-359.

Wait, J. R., and A. M. Conda (June 1961), A diffraction theory for LF sky wave propagation-an additional note, J. Geophys. Res. 66, No. 6, 1725-1729.

(Paper 68 D1-318) 